\title{
Dioscin protects against coronary heart disease by reducing oxidative stress and inflammation via Sirt1/Nrf2 and 338 MAPK pathways
}

\author{
BO YANG*, BIN XU*, HUA ZHAO*, YA-BIN WANG* , JIAN ZHANG, CHUAN-WEI LI, \\ QING WU, YU-KANG CAO, YANG LI and FENG CAO
}

Department of Cardiology, Chinese PLA General Hospital, Beijing 100853, P.R. China

Received March 20, 2017; Accepted January 11, 2018

DOI: $10.3892 / \mathrm{mmr} .2018 .9024$

\begin{abstract}
Cardiovascular diseases are common diseases in Sweden as in most countries. In 2016, 25,700 persons suffered from coronary heart disease (CHD) and $25 \%$ of these died within 28 days. The present study investigated whether dioscin may exert protective effects against CHD-induced heart apoptosis, oxidative stress and inflammation in a pig model and the potential underlying mechanisms. Adult pigs were used to establish a CHD model group and $80 \mathrm{mg} / \mathrm{kg}$ dioscin was administered for 4 weeks. Histological analysis and measurement of serum levels of heart injury markers demonstrated that $80 \mathrm{mg} / \mathrm{kg}$ dioscin markedly alleviated CHD, while left ventricular ejection fraction and left ventricular systolic internal diameter measurements indicated that $80 \mathrm{mg} / \mathrm{kg}$ dioscin also increased heart function in the CHD pig model. Furthermore, western blotting demonstrated that $80 \mathrm{mg} / \mathrm{kg}$ dioscin significantly reduced protein levels of apoptosis markers in the heart of CHD model pigs, including Bcl-2-associated $\mathrm{X}$ and caspase-3, potentially via the suppression of poly (ADP-ribose) polymerase 1 (PARP)/p53 expression. Additionally, the results of ELISA and western blotting demonstrated that $80 \mathrm{mg} / \mathrm{kg}$ dioscin may reduce oxidative stress and inflammation in CHD model pigs through the promotion of sirtuin 1 (Sirt1)/nuclear factor erythroid 2-related factor 2 (Nrf2) protein expression and the suppression of PARP/p53 and p38 mitogen-activated protein kinase (MAPK) expression. The results of the current study indicate that dioscin may protect against CHD by regulating oxidative stress and inflammation via Sirt1/Nrf2 and p38 MAPK pathways.
\end{abstract}

Correspondence to: Dr Bo Yang or Dr Feng Cao, Department of Cardiology, Chinese PLA General Hospital, 28 Fu-xing Road, Beijing 100853, P.R. China

E-mail: dryangb@aliyun.com

E-mail: fengcaofengrao@163.com

*Contributed equally

Key words: dioscin, coronary heart disease, inflammation, sirtuin 1/nuclear factor erythroid 2-related factor 2, p38 mitogen-activated protein kinase

\section{Introduction}

Atherosclerosis is a chronic pathophysiological process involving large and medium-sized vessels (1). It is considered to be the result of cholesterol accumulation in the artery over a long period of time. However, a series of landmark studies have allowed an increasingly clear understanding of the key role of inflammation in the genesis and development of atherosclerosis (2). Various types of immunocytes are the major components of early atherosclerotic plaques and effector molecules released by these immunocytes accelerate the plaque progression (3). Acute coronary syndrome is the manifestation of intra-plaque inflammation activation (3). Therefore, atherosclerosis is considered to be an inflammatory disease (3) and is the consequence of a combination of immune factors and metabolic risk factors (4). Atherosclerosis manifests as the genesis and development of atherosclerotic plaques within the vascular wall (4).

Interleukin (IL)-6 is a multifunctional circulating cytokine. Its major biological activities include inducing B cells to produce antibody and promoting cytotoxic $\mathrm{T}$ cell formation (5). Elevated levels of IL-6 are observed following cardiac surgery, which peak at 4-6 h (5). IL-6 has proinflammatory and anti-inflammatory effects and is an important acute phase reaction factor during the wound and repair process (6). IL-6 is able to activate neutrophils and also delay the phagocytosis of aging and non-functional neutrophils by phagocyte. Thus, it promotes an inflammatory environment (7). IL-6 has also been reported to promote the release of soluble tumor necrosis factor (TNF) receptors and IL-1 receptor (8), while another study demonstrated that IL- 6 weakened the effect of TNF- $\alpha$ and IL-1, thus exerting an anti-inflammatory effect. Excessive IL-6 release is a risk factor for patients (9).

The heart incessantly contracts and relaxes throughout the lifetime of an individual to drive the systemic blood circulation. In order to allow its function, the maintenance of sufficient blood, oxygen and nutrients is required (10). Energy is supplied to myocardial cells primarily through $\beta$-oxidation of fatty acids under aerobic conditions (11) and myocardial cells almost completely depend on aerobic metabolism to supply energy. Therefore, myocardial cells have a high dependency on oxygen. Myocardial cells have a high sensitivity to ischemia and anoxia; once myocardial cells suffer ischemia 
and anoxia, cell dysfunction, paramorphia and potentially death may occur (11).

Sirtuin 1 (Sirt1), also termed silent mating type information regulation 2 homolog 1, is a NAD2-dependent multifunctional transcription regulatory factor that is involved in the regulation of various signaling pathways that are involved in mammalian cell lifespan, glucose metabolism and insulin secretion (12). Previous studies have demonstrated that knock-out of Sirt1 aggravated myocardial ischemia-reperfusion injury in mice, indicating that SIRT1 may have a role in the protection against myocardial injury $(12,13)$.

The nuclear factor erythroid 2-related factor 2 (Nrf2)-antioxidant response element (ARE) pathway is the most important endogenous antioxidative stress pathway that has been identified at present. It is widely distributed in the cardiovascular system and upregulates the endogenous antioxidative system once activated, thereby reducing oxidative damage to the myocardium (14). In addition, as a receptor of oxidative stress, Nrf2 has important roles in the regulation of cellular oxidative stress and functions as a transcription factor of antioxidative stress (15). ARE has a unique induction mechanism to prevent against oxidative stress, and as a cis-acting element, is activated by various factors, including hydrogen peroxide, reactive oxygen species, electrophilic species and other xenobiotics, and induces the expression of antioxdative genes (16).

p38 mitogen-activated protein kinase (MAPK) is a type of tyrosine/threonine protein kinase with a molecular weight of $\sim 40-60 \mathrm{kDa}$; all members of the MAPK family are activated by dual phosphorylation of tyrosine and threonine (17). Tyrosine kinase receptors, G-protein-coupled receptors and ion channel-coupled receptors may initiate the phosphorylation of tyrosine and threonine sites on MAPKs through various intermediary links to activate p38 MAPK, and activated p38 MAPK subsequently translocates to its corresponding transcription factors and activates gene transcription, resulting in effects on cell proliferation, growth or apoptosis $(17,18)$. p38 MAPK is considered to be the focal point or common pathway of the transmission of various extracellular signals that lead to cell proliferation, hypertrophy and apoptosis, and participates in myocardial hypertrophy, proliferation and apoptosis induced by a various stimuli (19).

Dioscin is a type of steroid sapogenin synthesized naturally by plants and belongs to the group of spirostanols (20). As an important raw material for the synthesis of steroid hormones and steroidal contraceptives, dioscin has generally been used in the production of pregnenolone, progesterone, cortisol and other drugs (21). In the last several decades, the pharmacological effects of dioscin have been investigated extensively (20,21). Dioscin exhibits antineoplastic function and regulates blood lipids, prevents platelets from aggregation and promotes bile secretion; therefore, it is primarily employed to treat cardiovascular disease, encephalitis, skin diseases and tumors (22). The purpose of the current study was to investigate the potential protective effects of dioscin against coronary heart disease (CHD)-induced inflammation in a pig model and the underlying mechanisms.

\section{Materials and methods}

Experimental animals and groups. Chinese miniature pigs (male, 20-30 kg, 1-2 month) were acquired from the Institute

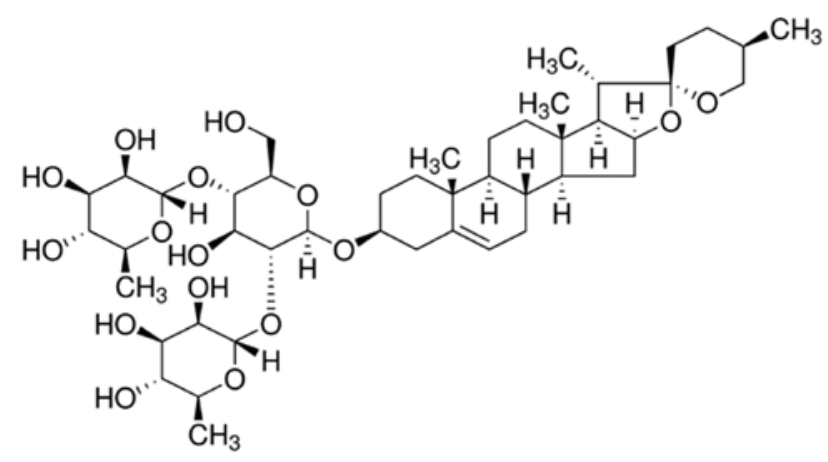

Figure 1. Chemical structure of dioscin.

of Laboratory Animal Science, Jining Medical University (Jining, China) and housed in $22-24^{\circ} \mathrm{C}, 55-60 \%$ humidity, $0.038 \% \mathrm{CO}_{2}$, fed a standard laboratory diet and water ad libitum with a $12 \mathrm{~h}$ light/dark cycle. A total of 26 pigs were randomly assigned into control (sham treatment, $n=6$ ), vehicle (CHD model, $\mathrm{n}=10$ ) and dioscin-treated (CHD model + dioscin, $n=10$ ) groups. Ethical approval for the present study was provided by the Chinese PLA General Hospital (Beijing, China). The chemical structure of dioscin is presented in Fig. 1.

CHD model induction and dioscin treatment. In the CHD model and dioscin-treated groups, pigs were fed with a high-fat diet for 4 weeks. Subsequently, pigs were intravenously administered with $30 \mathrm{mg} / \mathrm{kg}$ sodium pentobarbital (Sigma-Aldrich; Merck KGaA, Darmstadt, Germany) into the ear vein and the common carotid artery was separated and a $6 \mathrm{~F}$ arterial sheath tube was advanced and then the ligature was checked to ensure it was tight. The left anterior descending branch was accessed by a guide wire and the sacculus was merged into the left anterior descending branch and air pressure was maintained at 10-12 ATM for $30 \mathrm{sec}$ and this process was repeated 3 times with the pressure being maintained in between at 1-1.5 ATM. Subsequently, in CHD model and dioscin treated groups, pigs were fed with a high-fat diet for 4 weeks. In the dioscin-treated group, pigs intraperitoneal received with $80 \mathrm{mg} / \mathrm{kg} /$ every 3 days dioscin (Sigma-Aldrich; Merck KGaA) for 4 weeks. In control group, pigs were intravenously administered with $30 \mathrm{mg} / \mathrm{kg}$ sodium pentobarbital (Sigma-Aldrich; Merck $\mathrm{KGaA}$ ) into ear vein without induction of CHD.

Hematoxylin and eosin staining methods. After treatment with dioscin, tissue samples were washed with PBS and fixed with $4 \%$ paraformaldehyde for $24 \mathrm{~h}$ at room temperature. Tissue samples were embedded in paraffin and sectioned at $10 \mu \mathrm{M}$. Tissue samples were stained with hematoxylin and eosin staining for $15 \mathrm{~min}$ at room temperature. Tissue samples were observed using a LSM 780 NLO confocal microscope (Carl Zeiss, AG, Oberkochen, Germany).

Left ventricular ejection fraction (LVEF) and systolic internal diameter (LVIDS). Following treatment with dioscin, LVEF and LVIDs were analyzed by a S5-1 linear probe (iE33 xMatrix Ultrasound; Philips Healthcare, Andover, MA, USA). 

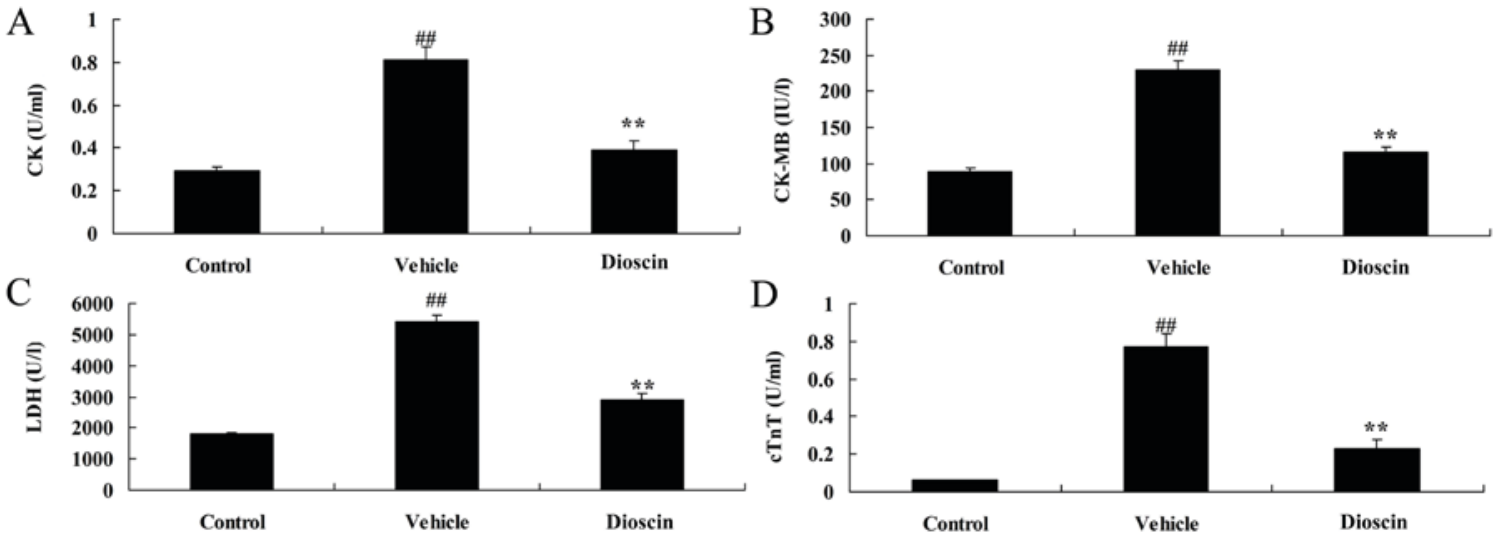

Figure 2. Dioscin reduces the levels of CK, CK-MB, LDH and cTnT in CHD model pigs. ELISA was performed following treatments to determine the levels of (A) CK, (B) CK-MB, (C) LDH and (D) cTnT in the serum of pigs. ${ }^{\# \prime} \mathrm{P}<0.01$ vs. control group; ${ }^{* *} \mathrm{P}<0.01$ vs. vehicle group. CK, creatine kinase; $\mathrm{LDH}$, lactate dehydrogenase; cTnT, cardiac troponin T; CHD, coronary heart disease; control, sham group; vehicle, CHD model group; dioscin, CHD model + dioscin group.

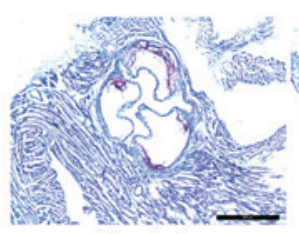

Control

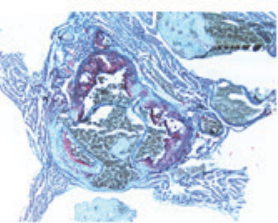

Vehicle

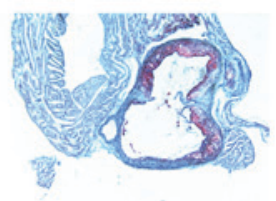

Dioscin
Figure 3. Dioscin reduces the presence of arterial plaques in a CHD pig model. (Magnification, x20), Control, sham group; vehicle, CHD model group; dioscin, CHD model + dioscin group.

Determination of serum levels of heart injury and inflammatory markers using ELISA kits. Following treatment with dioscin, serum samples from each group were obtained from whole blood samples by centrifugation at 2,000 $\mathrm{x}$ g for $10 \mathrm{~min}$ at $4{ }^{\circ} \mathrm{C}$. The levels of creatine kinase (CK; cat. no. A032), CK-MB (cat. no. H197), lactate dehydrogenase (LDH; cat. no. A020-2), cardiac troponin T (cTnT; cat. no. H149-4), TNF- $\alpha$ (cat. no. H052), IL-1 $\beta$ (cat. no. H002), IL-6 (cat. no. H007) and IL-18 (cat. no. H015) were measured using respective ELISA kits (Nanjing Jiancheng Bioengineering Institute, Nanjing, China). SOD (cat. no. A001-1-1), MDA (cat. no. A003-1), CAT (cat. no. A007-1-1) and GSH (cat. no. A006-2) levels were measured using commercial kit.

Western blot analysis. Following treatment with dioscin for 4 weeks, proteins were extracted from coronary tissue samples using radioimmunoprecipitation assay lysis buffer and protein concentration was measured using a BCA protein assay kit (both Beyotime Institute of Biotechnology, Haimen, China) following centrifugation at $12,000 \mathrm{x}$ g for $10 \mathrm{~min}$ at $4^{\circ} \mathrm{C}$. Total protein $(50 \mu \mathrm{g})$ was separated on $8-12 \%$ SDS-PAGE gels and transferred to polyvinylidene difluoride membranes (EMD Millipore, Billerica, MA, USA). The membranes were subsequently blocked with $5 \%$ non-fat milk for $1 \mathrm{~h}$ at $37^{\circ} \mathrm{C}$ followed by incubation with $\operatorname{Bax}(1: 1,000$; cat. no. sc-6236), Caspase-3 (1:1,000; cat. no. sc-98785), PARP (1:1,000; cat. no. sc-7150), p53 (1:1,000; cat.no. sc-6243), Sirt1 (1:1,000, cat.no. sc-15404), Nrf2 (1:1,000; cat. no. sc-722), p38 (1:1,000; sc-728) and p-p38 (1:1,000; sc-17852-R) and GAPDH (cat. no. sc-25778;
1:500; all Santa Cruz Biotechnology, Inc.) at $4^{\circ} \mathrm{C}$ overnight. The membrane was washed with TBS-0.1\% Tween-20 and incubated with horseradish peroxidase-conjugated secondary antibody (cat. no. sc-2004; 1:5,000; Santa Cruz Biotechnology, Inc.) for $1 \mathrm{~h}$ at $37^{\circ} \mathrm{C}$. Subsequently, the membrane was stained with ECL Plus (Beyotime Institute of Biotechnology) and analyzed using Image_Lab_3.0 (Bio-Rad Laboratories, Inc.).

Statistical analysis. Data are presented as the mean \pm standard error of the mean $(n=6)$. Data were analyzed by SPSS 17.0 software (SPSS, Inc., Chicago, IL, USA) and were analyzed by one-way analysis of variance and the post-hoc Bonferroni test. $\mathrm{P}<0.05$ was considered to indicate a statistically significant difference.

\section{Results}

Dioscin protects against CHD in a pig model. The present study investigated whether dioscin may exert protective effects against CHD in a pig model. The results of ELISA demonstrated that the serum levels of heart injury markers CK, CK-MB, LDH and cTnT in the CHD model group were significantly higher compared with the control group (Fig. 2). Following treatment with $80 \mathrm{mg} / \mathrm{kg}$ dioscin, the serum levels of CK, CK-MB, LDH and cTnT were significantly lower compared with the CHD model group (Fig. 2). Furthermore, hematoxylin and eosin staining demonstrated that there was a higher number of arterial plaques in the CHD model group compared with the control group. However, dioscin $(80 \mathrm{mg} / \mathrm{kg}$ ) markedly prevented the formation of arterial plaques in CHD pigs, compared with the CHD model group (Fig. 3).

Dioscin improves heart function in CHD model pigs. To determine whether dioscin protects heart function in CHD model pigs, LVEF and LVIDs were measured in each group. In the CHD model group, the LVEF was lower compared with the control group (Fig. 4A). However, CHD-induced reduction of LVEF was significantly reversed by $80 \mathrm{mg} / \mathrm{kg}$ dioscin (Fig. 4A). By contrast, LVIDs was higher in the CHD model group compared with the control group and dioscin $(80 \mathrm{mg} / \mathrm{kg})$ 
significantly reduced the LVIDs in CHD pigs, compared with the CHD model group (Fig. 4B).

Dioscin reduces oxidative stress and the levels of inflammation in a CHD pig model. The results demonstrated that the levels of superoxide dismutase (SOD), catalase (CAT) and glutathione (GSH) were significantly decreased, while malondialdehyde (MDA) levels were significantly increased, in the CHD model group compared with the control group (Fig. 5). Treatment with dioscin $(80 \mathrm{mg} / \mathrm{kg})$ significantly increased SOD, CAT and GSH levels, and inhibited MDA levels, in CHD pigs, compared with the CHD model group (Fig. 5). Furthermore, TNF- $\alpha$, IL-1 $\beta$, IL- 6 and IL-18 levels were significantly increased in the CHD model group compared with the control group, and these CHD-induced increases in TNF- $\alpha$, IL-1 $\beta$, IL-6 and IL-18 were significantly reduced by treatment with $80 \mathrm{mg} / \mathrm{kg}$ dioscin (Fig. 6).

Dioscin inhibits apoptosis in the heart of CHD model pigs. Compared with the control group, the protein expression of caspase- 3 and Bcl-2-associated X (Bax) in coronary tissues was significantly increased in the CHD model group (Fig. 7). However, $80 \mathrm{mg} / \mathrm{kg}$ dioscin significantly suppressed the CHD-induced increases in the expression of caspase- 3 and Bax (Fig. 7).

Dioscin suppresses poly (ADP-ribose) polymerase 1 (PARP) and 553 protein expression in CHD model pigs. To investigate the signal transduction mechanisms of dioscin-mediated protection against heart cell apoptosis in CHD observed in the present study, the alterations in PARP and $\mathrm{p} 53$ protein expression were also investigated. In the CHD model group, PARP and p53 protein expression were significantly increased compared with the control group (Fig. 8). However, dioscin significantly suppressed PARP and p53 protein expression compared with the CHD model group (Fig. 8). These results indicated that the protective effects of dioscin on heart cell apoptosis in CHD may be mediated via PARP and $\mathrm{p} 53$ proteins.

Dioscin induces Sirt1/Nrf2 and suppresses p38 MAPK pathways in a CHD pig model. To investigate the roles of Sirt1/Nrf2 and p38 MAPK pathways in dioscin-mediated protection against oxidative stress and inflammation in CHD, the protein expression of components of Sirt1/Nrf2 and p38 MAPK pathways was measured. As demonstrated in Fig. 9, Sirt1 and Nrf2 protein expression was significantly suppressed, while phosphorylated (p)-p38 MAPK protein expression was significantly induced, in the CHD model group compared with the control group. However, $80 \mathrm{mg} / \mathrm{kg}$ dioscin significantly induced Sirt1 and Nrf2 protein expression and suppressed p-p38 MAPK protein expression in CHD model pigs, compared with the CHD model group (Fig. 9). These results indicated that dioscin may reduce oxidative stress and inflammation in CHD through activation of Sirt1/Nrf2 and suppression of p38 MAPK pathways.

\section{Discussion}

Research concerning atherosclerosis has been increasingly intensive. Thus, the importance of inflammation in the genesis and development of atherosclerosis has become increasingly clear. Inflammation was reported to have an important role in acute coronary syndrome (23) and is also a factor associated with the genesis and development of atherosclerotic plaques (24). At present, the concept that atherosclerosis is an inflammatory disease has been widely accepted. Atherosclerosis is the result of a combination of immune factors and metabolic risk factors and manifests as the genesis and development of atherosclerotic plaques within the vascular wall (25). The results of the present study demonstrated that dioscin reduced the serum levels of CK, CK-MB, LDH and cTnT, increased the LVEF and reduced the LVIDs in CHD model pigs. In addition, Qin et al (26) demonstrated that Dioscin prevents the mitochondrial apoptosis and attenuates oxidative stress in cardiac $\mathrm{H} 9 \mathrm{c} 2$ cells.

ROS is a type of metabolic substance that is generated from oxygen (27). There are various types of active oxygen and oxygen radicals are the major type, which include hydroxyl radicals, superoxide anion, nitric oxide and certain non-free radicals. A certain level of active oxygen is required for the maintenance of normal life and usually exists in a dynamic equilibrium in the body at a normal level (10). The physiological functions of active oxygen include participation in electron transfer and metabolic processes within the body (28). When stress is induced in the body by various conditions, including ischemia, anoxia, ion radiation and chemical reagents, the generation of active oxygen forms may be induced in body, which may lead to an imbalance between the levels of oxidative and antioxidative factors, and subsequent cell damage (28). In the current study, dioscin significantly increased SOD, CAT and GSH levels and decreased MDA levels in CHD model pigs. In addition, Zhao et al (29) suggested that dioscin alleviated doxorubicin-induced cardiotoxicity through modulating miR-140-5p-mediated myocardial oxidative stress.

Sirtl was reported to alleviate oxidative stress and inflammatory reactions, activate the autophagy of myocardial cells and inhibit the apoptosis of myocardial cells induced by ischemia-reperfusion to protect the myocardium (30). Furthermore, in a study concerning the effect of Sirt1 on the expression of endoplasmic reticulum stress-associated proteins during myocardial ischemia-reperfusion, it was demonstrated that the mRNA and protein expression levels of endoplasmic reticulum stress-associated genes (glucose-regulated protein $78 \mathrm{kDa}$, caspase-3 and DNA damage-inducible transcript 3) decreased with the activation of Sirt1 and increased with the inhibition of Sirt1 (31). Therefore, Sirt1 was indicated to protect the myocardium from ischemia-reperfusion by inhibiting the expression of endoplasmic reticulum stress-associated proteins (31). The results of the present study indicated that dioscin significantly increased the expression of Sirtl protein in CHD model pigs.

Nrf2 and the downstream antioxidant genes that it regulates participate in the protective mechanism against myocardial ischemia-reperfusion injury (32). Previous studies demonstrated that the basal expression of antioxidants and two-phase enzyme in Nrf2 knockout mice was markedly lower compared with wild-type mice, with sensitivity to ROS-induced cytotoxicity increased, indicating that antioxidant genes were vital to protecting against myocardial injury and the expression 
A

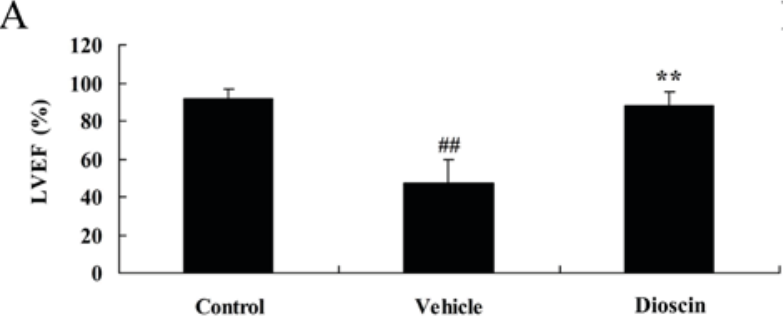

B

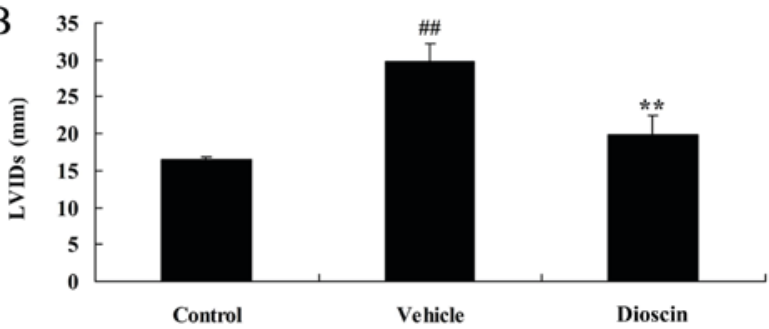

Figure 4. Dioscin alters the LVEF and LVIDs in CHD model pigs. Effects of dioscin on the (A) LVEF and (B) LVIDs of CHD model pigs. ${ }^{\sharp \#} \mathrm{P}<0.01$ vs. control group; ${ }^{* *} \mathrm{P}<0.01$ vs. vehicle group. LVEF, left ventricular ejection fraction; LVIDs, left ventricular systolic internal diameter; CHD, coronary heart disease; control, control group; vehicle, CHD model group; dioscin, CHD model + dioscin group.

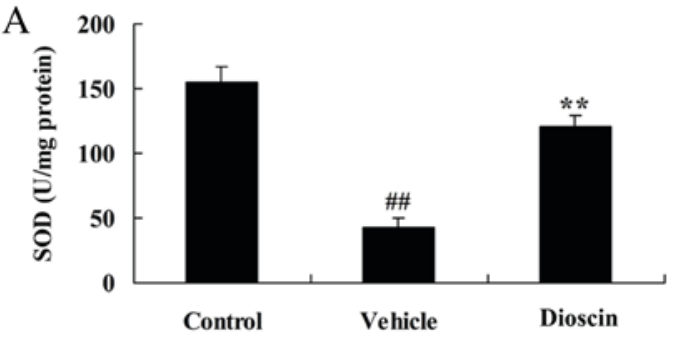

$\mathrm{C}$

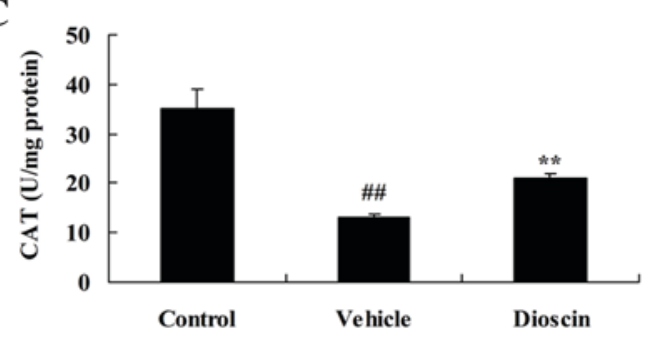

B

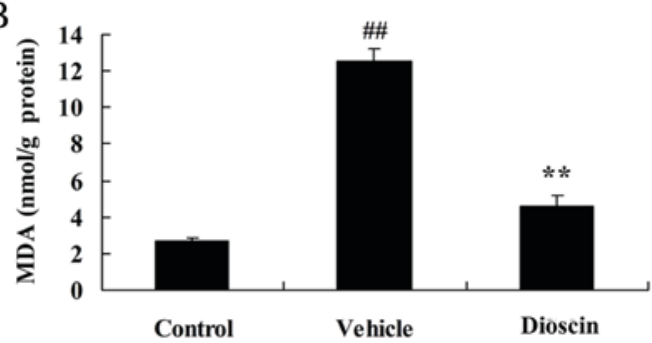

$\mathrm{D}$

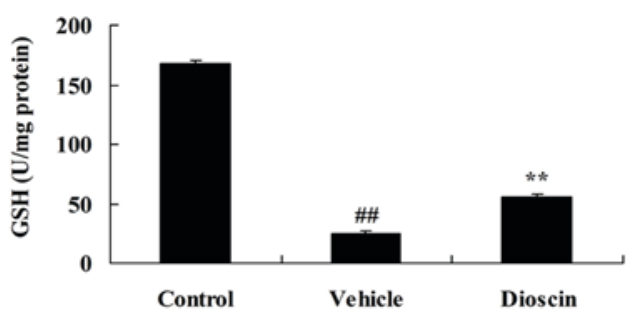

Figure 5. Dioscin reduces oxidative stress in CHD model pigs. The effect of dioscin on the serum levels of (A) SOD, (B) MDA, (C) CAT and (D) GSH in CHD model pigs was determined by ELISA. ${ }^{\# \#} \mathrm{P}<0.01$ vs. control group; ${ }^{* *} \mathrm{P}<0.01$ vs. vehicle group. CHD, coronary heart disease; SOD, superoxide dismutase; MDA, malondialdehyde; CAT, catalase; GSH, glutathione; control, sham group; vehicle, CHD model group; dioscin, CHD model + dioscin group.

A

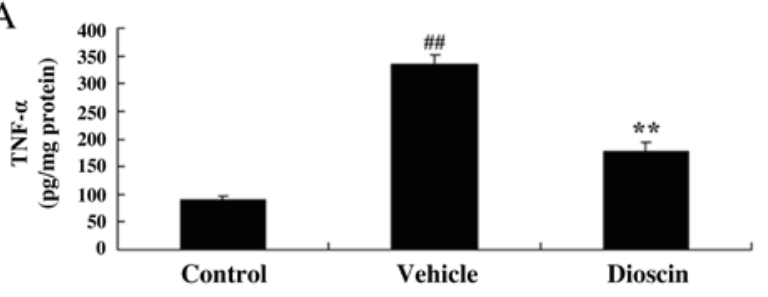

$\mathrm{C}$

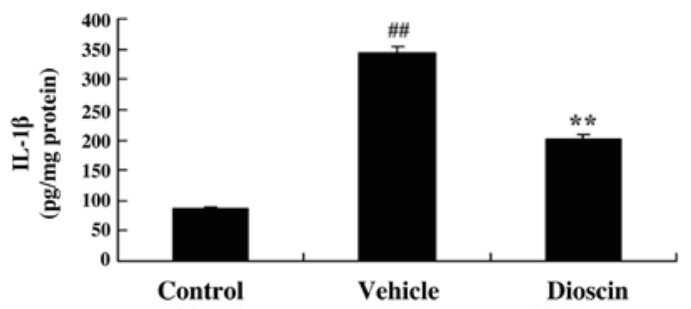

B

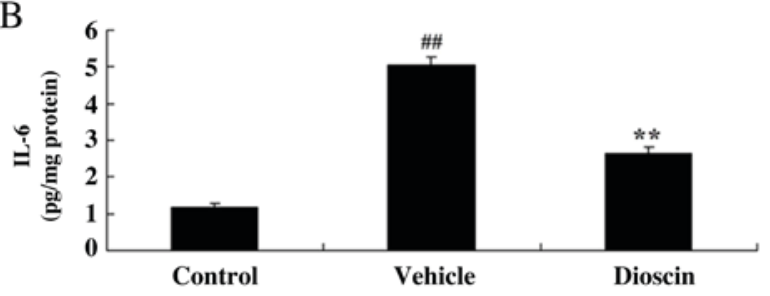

D

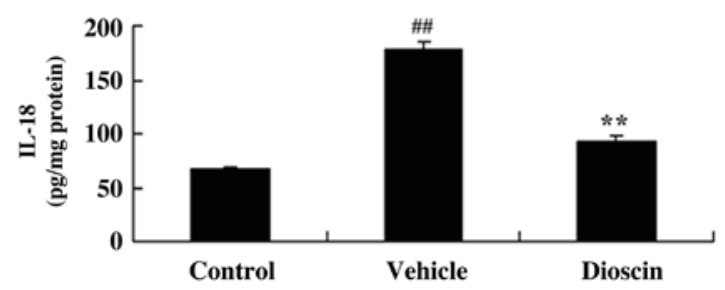

Figure 6. Dioscin reduces the levels of inflammatory cytokines in CHD model pigs. ELISA was performed to determine the effect of dioscin on the serum levels of (A) TNF- $\alpha$, (B) IL-6, (C) IL-1 $\beta$ and (D) IL-18 in CHD model pigs. ${ }^{\# \#} \mathrm{P}<0.01$ vs. control group; ${ }^{* *} \mathrm{P}<0.01$ vs. vehicle group. CHD, coronary heart disease; TNF, tumor necrosis factor; IL, interleukin; control, sham group; vehicle, CHD model group; dioscin, CHD model + dioscin group.

of Nrf2 was involved in the expression and induction of antioxidants. In addition, knockout of Nrf2 inhibited the ability of fibroblasts to fight against injury induced by ROS $(33,34)$. Importantly, the present study demonstrated that dioscin significantly increased Nrf2 protein expression in the heart of CHD model pigs, and the results indicated that the effects of dioscin may be mediated by Sirt1/Nrf2 to protect against CHD in pigs. Additionally, Gu et al (22) demonstrated that 

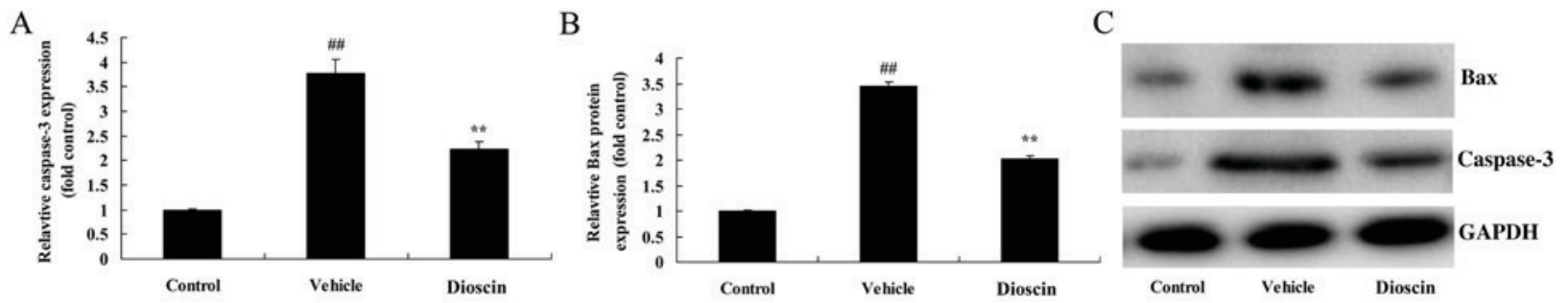

Figure 7. Dioscin reduces the protein levels of caspase-3 and Bax in CHD model pigs. Densitometric analysis of western blotting results demonstrated that dioscin reduced the protein levels of (A) caspase-3 and (B) Bax in CHD model pigs. (C) Representative western blot bands for caspase-3, Bax and GAPDH in each of the three groups. ${ }^{\# \#} \mathrm{P}<0.01$ vs. control group; ${ }^{* *} \mathrm{P}<0.01$ vs. vehicle group. Bax, Bcl-2-associated $\mathrm{X} ; \mathrm{CHD}$, coronary heart disease; control, sham group; vehicle, CHD model group; dioscin, CHD model + dioscin group.

A

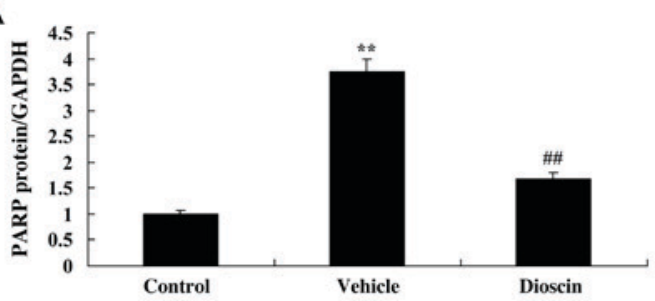

B

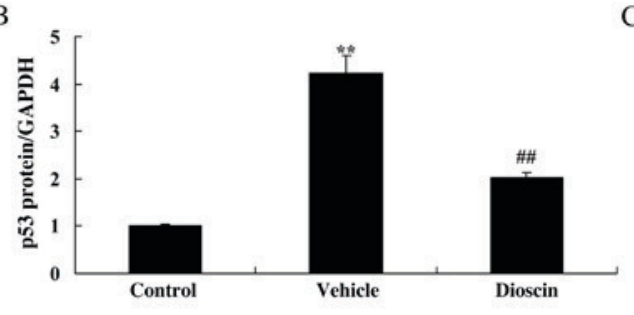

C

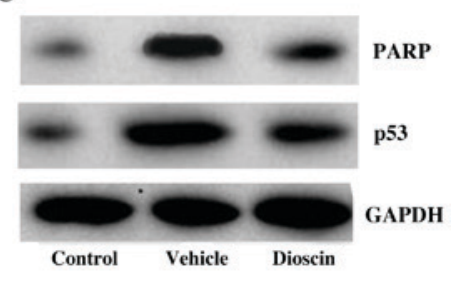

Figure 8. Dioscin reduces PARP and p53 protein expression in CHD model pigs. Densitometric analysis of western blotting results demonstrated that dioscin reduced the protein levels of (A) PARP and (B) p53 in CHD model pigs. (C) Representative western blot bands for PARP, p53 and GAPDH in each of the three groups. ${ }^{\# \#} \mathrm{P}<0.01$ vs. control group; ${ }^{* *} \mathrm{P}<0.01$ vs. vehicle group. PARP, poly (ADP-ribose) polymerase 1; CHD, coronary heart disease; control, sham group; vehicle, CHD model group; dioscin, CHD model + dioscin group.

A

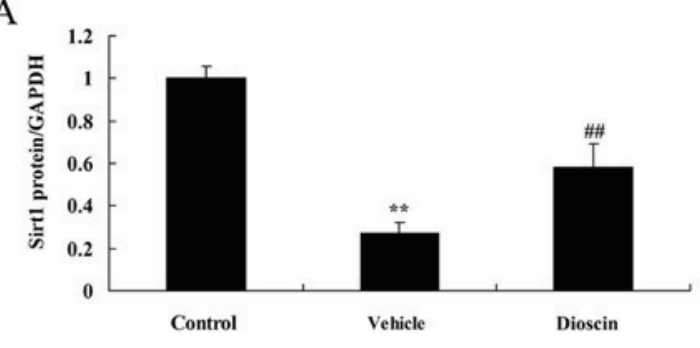

$\mathrm{C}$

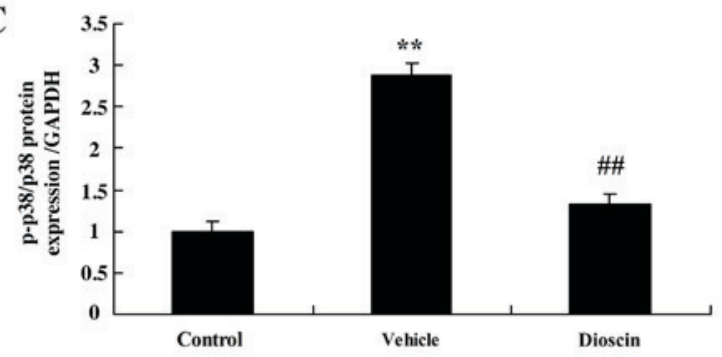

$\mathrm{B}$

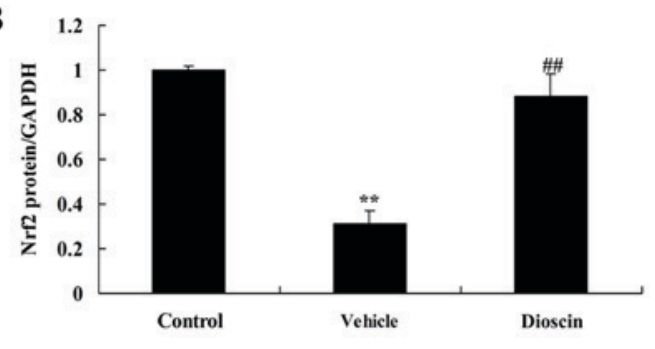

$\mathrm{D}$

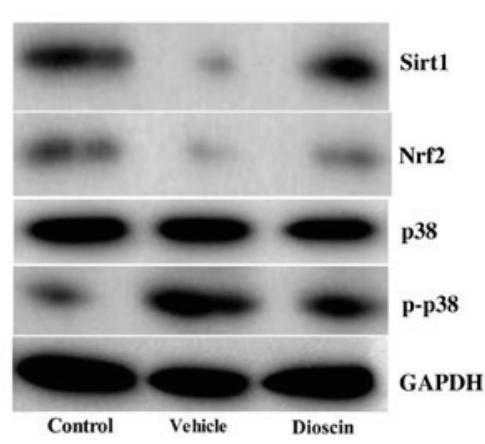

Figure 9. Dioscin activates Sirt1/Nrf2 and inhibits p38 MAPK pathways. Densitometric analysis of western blotting results was performed to determine the effect of dioscin on the protein levels of (A) Sirt1, (B) Nrf2 and (C) p-p38 MAPK in coronary heart disease model pigs. (D) Representative western blot bands for Sirt1, Nrf2, p38 and p-p38 MAPK, and GAPDH in each of the three groups. ${ }^{\# \#} \mathrm{P}<0.01$ vs. control group; ${ }^{* *} \mathrm{P}<0.01$ vs. vehicle group. Sirt1, sirtuin 1; Nrf2, nuclear factor erythroid 2-related factor 2; MAPK, mitogen-activated protein kinase; p-, phosphorylated-; control, sham group; vehicle, CHD model group; dioscin, CHD model + dioscin group.

dioscin alleviated hepatic fibrosis through the Sirt1/Nrf2 and p38 MAPK pathways.

As an important member of the MAPK family, p38 MAPK has been reported to be involved in the ischemia, reperfusion and apoptosis of myocardial cells in a previous study (35). Numerous stimuli may lead to the activation of p38 MAPK and activated p38 MAPK has been implicated in the regulation of myocardial cell apoptosis (35). Myocardial ischemia-reperfusion has been reported to initiate the stress-activated protein kinase pathway and MAPK pathway in cells, and these signal pathways were demonstrated to be closely associated with calcineurin (36). The activity of 
calcineurin was reported to be enhanced during myocardial ischemia-reperfusion and participated in myocardial apoptosis induced by ischemia-reperfusion; p38 MAPK are important signal pathways that mediate myocardial apoptosis (17). The results of the present study demonstrated that dioscin significantly suppressed p-p38 MAPK protein expression in CHD model pigs, indicating that p38 MAPK signaling pathways may also be involved in the effects of dioscin in CHD model pigs. Wang et al (37) demonstrated that dioscin induced the apoptosis of HL-60 cells through activation of p38 MAPK and c-Jun N-terminal kinase pathways.

In conclusion, the results of the current study demonstrated that dioscin reduced the levels of CK, CK-MB, LDH and cTnT, increased the LVEF and inhibited the LVIDs CHD model pigs through anti-inflammatory and antioxidative effects. Furthermore, the results of further experiments indicated that the protective effects of dioscin on CHD in pigs may be associated with the Sirt1/Nrf2 and p38 MAPK pathways, and dioscin maybe a novel possible drug for CHD in future research.

\section{Acknowledgements}

Not applicable.

\section{Funding}

The present study was partially supported by the National Natural Science Foundation of China (grant no. 81570272, to Dr Bo Yang), the Beijing Natural Science Foundation (grant no. 7132227, to Dr Bo Yang), National Science Foundation of China (grant no. 61471064, GX Kang and B Yang), the Nova Programme from Beijing Municipal Science and Technology Commission (grant no. Z141107001814113-XXHZ201401, to Dr Bo Yang) and the Discovery Foundation from The Chinese Medical Doctor Association (grant no. DFCMDA201311, to Dr Bo Yang).

\section{Availability of data and materials}

The datasets used and/or analyzed during the current study are available from the corresponding author on reasonable request.

\section{Authors' contributions}

BY designed the study; BX, HZ, YBW, JZ, CWL, QW, YKC, YL and FC performed the experiments; BY and FC analyzed the data; BY wrote the manuscript.

\section{Ethics approval and consent to participate}

Ethical approval for the present study was provided by the Chinese PLA General Hospital (Beijing, China).

\section{Consent for publication}

Not applicable.

\section{Competing interests}

All authors declared that they have no competing of interests.

\section{References}

1. Andersson C, Shilane D, Go AS, Chang TI, Kazi D, Solomon MD, Boothroyd DB and Hlatky MA: Beta-blocker therapy and cardiac events among patients with newly diagnosed coronary heart disease. J Am Coll Cardiol 64: 247-252, 2014.

2. Strissel KJ, Denis GV and Nikolajczyk BS: Immune regulators of inflammation in obesity-associated type 2 diabetes and coronary artery disease. Curr Opin Endocrinol Diabetes Obes 21: 330-338, 2014.

3. Hakeem A, Bhatti S and Chang SM: Screening and risk stratification of coronary artery disease in end-stage renal disease. JACC Cardiovasc Imaging 7: 715-728, 2014.

4. Alizade E, Avci A, Acar G, Açar G, Fidan S, Öcal L, Bulut M, Tellice M, Akçakoyun M, Pala S and Esen AM: The relationship between rheumatoid factor levels and coronary artery lesion complexity and severity in patients with stable coronary artery disease. Postepy Kardiol Interwencyjnej 11: 26-31, 2015.

5. Tan F, Chen Y, Yuan D, Gong C, Li X and Zhou S: Dexmedetomidine protects against acute kidney injury through downregulating inflammatory reactions in endotoxemia rats. Biomed Rep 3: 365-370, 2015.

6. Meyer S, Neeff H, Thomusch O, Strate T, Tittelbach-Helmrich D, Hopt UT and von Dobschuetz E: Everolimus improves microcirculatory derangements in experimental postischemic pancreatitis modulating the expression of vascular endothelial growth factor, Interleukin 6, and toll-like receptor 4. Pancreas 44: 1245-1251, 2015.

7. Correia GD, Wooi Ng K, Wijeyesekera A, Gala-Peralta S, Williams R, MacCarthy-Morrogh S, Jiménez B, Inwald D, Macrae D, Frost G, et al: Metabolic profiling of children undergoing surgery for congenital heart disease. Crit Care Med 43: 1467-1476, 2015.

8. Yin YW, Li JC, Zhang M, Wang JZ, Li BH, Liu Y, Liao SQ, Zhang MJ, Gao CY and Zhang LL: Influence of interleukin-6 gene $-174 \mathrm{G}>\mathrm{C}$ polymorphism on development of atherosclerosis: A meta-analysis of 50 studies involving 33,514 subjects. Gene 529: 94-103, 2013.

9. Sakthivel KM and Guruvayoorappan C: Acacia ferruginea inhibits tumor progression by regulating inflammatory mediators-(TNF-a, iNOS, COX-2, IL-1 $\beta$, IL-6, IFN- $\gamma$, IL-2, GM-CSF) and pro-angiogenic growth factor- VEGF. Asian Pac J Cancer Prev 14: 3909-3919, 2013.

10. Somacal S, Figueiredo CG, Quatrin A, Ruviaro AR, Conte L, Augusti PR, Roehrs M, Denardin IT, Kasten J, da Veiga ML, et al: The antiatherogenic effect of bixin in hypercholesterolemic rabbits is associated to the improvement of lipid profile and to its antioxidant and anti-inflammatory effects. Mol Cell Biochem 403: 243-253, 2015.

11. Badalzadeh R, Mohammadi M, Yousefi B, Farajnia S, Najafi M and Mohammadi S: Involvement of glycogen synthase kinase- $3 \beta$ and oxidation status in the loss of cardioprotection by postconditioning in chronic diabetic male rats. Adv Pharm Bull 5: 321-327, 2015.

12. Fleming DS and Miller LC: Identification of small non-coding RNA classes expressed in swine whole blood during HP-PRRSV infection. Virology 517: 56-61, 2018.

13. Saito S, Thuc LC, Teshima Y, Nakada C, Nishio S, Kondo H, Fukui A, Abe I, Ebata Y, Saikawa T, et al: Glucose fluctuations aggravate cardiac susceptibility to ischemia/reperfusion injury by modulating microRNAs expression. Circ J 80: 186-195, 2016.

14. Wang $\mathrm{J}, \mathrm{Hu} \mathrm{X}$ and Jiang $\mathrm{H}$ : ERS-PERK signaling pathway-mediated Nrf2/ARE-HO-1 axis: A novel therapeutic target for attenuating myocardial ischemia and reperfusion injury. Int J Cardiol 203: 779-780, 2016.

15. Mleczko AM and Bąkowska-Żywicka K: When small RNAs become smaller: Emerging functions of snoRNAs and their derivatives. Acta Biochim Pol 63: 601-607, 2016.

16. Li W, Wu M, Tang L, Pan Y, Liu Z, Zeng C, Wang J, Wei T and Liang G: Novel curcumin analogue 14 p protects against myocardial ischemia reperfusion injury through Nrf2-activating anti-oxidative activity. Toxicol Appl Pharmacol 282: 175-183, 2015.

17. Liao J, Yu L, Mei Y, Guarnera M, Shen J, Li R, Liu Z and Jiang F: Small nucleolar RNA signatures as biomarkers for non-small-cell lung cancer. Mol Cancer 9: 198, 2010.

18. Ravo M, Cordella A, Rinaldi A, Bruno G, Alexandrova E, Saggese P,Nassa G, Giurato G, Tarallo R, Marchese G, et al: Small non-coding RNA deregulation in endometrial carcinogenesis. Oncotarget 6: 4677-4691, 2015. 
19. Langhendries JL, Nicolas E, Doumont G, Goldman S and Lafontaine DL: The human box C/D snoRNAs U3 and U8 are required for pre-rRNA processing and tumorigenesis. Oncotarget 7: 59519-59534, 2016.

20. Zhang W, Yin L, Tao X, Xu L, Zheng L, Han X, Xu Y, Wang C and Peng J: Dioscin alleviates dimethylnitrosamine-induced acute liver injury through regulating apoptosis, oxidative stress and inflammation. Environ Toxicol Pharmacol 45: 193-201, 2016.

21. Lu B, Xu Y, Xu L, Cong X, Yin L, Li H and Peng J: Mechanism investigation of dioscin against $\mathrm{CCl}$-induced acute liver damage in mice. Environ Toxicol Pharmacol 34: 127-135, 2012.

22. Gu L, Tao X, Xu Y, Han X, Qi Y, Xu L, Yin L and Peng J: Dioscin alleviates BDL- and DMN-induced hepatic fibrosis via Sirt1/Nrf2-mediated inhibition of p38 MAPK pathway. Toxicol Appl Pharmacol 292: 19-29, 2016.

23. Konstanty-Kalandyk J, Piatek J, Miszalski-Jamka T, Rudziński P, Walter Z, Bartuś K, Urbańczyk-Zawadzka M and Sadowski J: The combined use of transmyocardial laser revascularisation and intramyocardial injection of bone-marrow derived stem cells in patients with end-stage coronary artery disease: One year follow-up. Kardiol Pol 71: 485-492, 2013.

24. Tahara N, Tahara A, Narula J and Imaizumi T: Statin therapy resolves coronary artery inflammation. JACC Cardiovase Imaging 6: 1119-1120, 2013.

25. Gupta GK, Agrawal T, DelCore MG, Mohiuddin SM and Agrawal DK: Vitamin D deficiency induces cardiac hypertrophy and inflammation in epicardial adipose tissue in hypercholesterolemic swine. Exp Mol Pathol 93: 82-90, 2012.

26. Qin J, Kang Y, Xu Z, Zang C, Fang B and Liu X: Dioscin prevents the mitochondrial apoptosis and attenuates oxidative stress in cardiac H9c2 cells. Drug Res (Stuttg) 64: 47-52, 2014.

27. Sartore G, Seraglia R, Burlina S, Bolis A, Marin R, Manzato E, Ragazzi E, Traldi P and Lapolla A: High-density lipoprotein oxidation in type 2 diabetic patients and young patients with premature myocardial infarction. Nutr Metab Cardiovasc Dis 25 418-425, 2015.

28. Mentese U, Dogan OV, Turan I, Usta S, Dogan E, Mentese SO, Demir S, Ozer T, Aykan AC and Alver A: Oxidant-antioxidant balance during on-pump coronary artery bypass grafting. ScientificWorldJournal 2014: 263058, 2014.
29. Zhao L, Tao X, Qi Y, Xu L, Yin L and Peng J: Protective effect of dioscin against doxorubicin-induced cardiotoxicity via adjusting microRNA-140-5p-mediated myocardial oxidative stress. Redox Biol 16: 189-198, 2018.

30. Ge J, Wu XM, Yang XT, Gao JM, Wang F and Ye KF: Role of long non-coding RNA SNHG1 in occurrence and progression of ovarian carcinoma. Eur Rev Med Pharmacol Sci 22: 329-335, 2018.

31. Ren J, Yang Y, Xue J, Xi Z, Hu L, Pan SJ and Sun Q: Long noncoding RNA SNHG7 promotes the progression and growth of glioblastoma via inhibition of miR-5095. Biochem Biophys Res Commun 496: 712-718, 2018.

32. Bachellerie JP, Nicoloso M, Qu LH, Michot B Caizergues-Ferrer M, Cavaille J and Renalier MH: Novel intron-encoded small nucleolar RNAs with long sequence complementarities to mature rRNAs involved in ribosome biogenesis. Biochem Cell Biol 73: 835-843, 1995.

33. Wang J, Cao L, Wu J and Wang Q: Long non-coding RNA SNHG1 regulates NOB1 expression by sponging miR-326 and promotes tumorigenesis in osteosarcoma. Int J Oncol 52: 77-88, 2018.

34. Mei YP, Liao JP, Shen J, Yu L, Liu BL, Liu L, Li RY, Ji L, Dorsey SG, Jiang ZR, et al: Small nucleolar RNA 42 acts as an oncogene in lung tumorigenesis. Oncogene 31: 2794-2804, 2012.

35. Koduru SV, Tiwari AK, Leberfinger A, Hazard SW, Kawasawa YI, Mahajan M and Ravnic DJ: A comprehensive NGS data analysis of differentially regulated miRNAs, piRNAs, lncRNAs and sn/snoRNAs in triple negative breast cancer. J Cancer 8: 578-596, 2017.

36. Gao L, Ma J, Mannoor K, Guarnera MA, Shetty A, Zhan M, Xing L, Stass SA and Jiang F: Genome-wide small nucleolar RNA expression analysis of lung cancer by next-generation deep sequencing. Int J Cancer 136: E623-E629, 2015.

37. Wang Y, He QY and Chiu JF: Dioscin induced activation of $\mathrm{p} 38$ MAPK and JNK via mitochondrial pathway in HL-60 cell line. Eur J Pharmacol 735: 52-58, 2014. 